\title{
An abundance study of the B-type targets for the asteroseismology programme of the CoRoT mission
}

\author{
T. Morel, ${ }^{1,2}$ C. Aerts ${ }^{1,3}$ \\ ${ }^{1}$ Katholieke Universiteit Leuven, Instituut voor Sterrenkunde, B-3001 Leuven, Belgium \\ ${ }^{2}$ European Space Agency (ESA) postdoctoral external fellow \\ ${ }^{3}$ Department of Astrophysics, University of Nijmegen, 6500 GL Nijmegen, The Netherlands
}

\begin{abstract}
We present the very first results of a project aimed at deriving the detailed chemical composition of the B-type stars in the eyes of CoRoT, focusing here on the two primary targets HD 170580 and HD 180642.
\end{abstract}

\section{Setting the stage for the CoRoT observations of B-type stars}

The French-European satellite CoRoT will soon monitor with extremely high photometric precision and time sampling a vast number of B-type stars during the course of its asteroseismology programme. Accurate estimates of the fundamental parameters and metallicity of these targets is essential to constrain the theoretical models and to allow for a proper interpretation of the space data. We are engaged in a project whose objectives are a homogeneous determination of both the atmospheric parameters (Lefever et al., in prep.) and the chemical composition (Morel et al., in prep.) of all the B stars in the eyes of CoRoT with high-resolution optical spectra secured as part of the ground-based preparatory campaign (GAUDI database; Solano et al. 2005). Most key chemical elements significantly contributing to the metallicity are considered: $\mathrm{C}, \mathrm{N}, \mathrm{O}, \mathrm{Mg}, \mathrm{Al}, \mathrm{Si}, \mathrm{S}$ and $\mathrm{Fe}$. Only $\mathrm{Ne}$ is missing at this stage, but work is currently underway to determine the abundance of this important element in the context of asteroseismological studies.

\section{First abundance results for two primary B-type targets}

Here we report on our analysis of two primary targets which will be observed during the first long runs of the mission: HD 170580 (B2 V) and HD 180642 (B1.5 II-III). The latter is a largeamplitude pulsator with three frequencies already detected from ground-based photometric data (Aerts et al., in prep.). The FEROS spectrum of HD 170580 is extracted from the GAUDI database, while the analysis of HD 180642 is based on 11 FEROS spectra taken in May 2006 with the ESO/MPI 2.2m Telescope at la Silla. See Morel et al. (2006) for details on the methodology used to derive the NLTE chemical abundances. Table 1 presents the results which can be compared with literature data for early B-type dwarfs in the solar vicinity (Daflon \& Cunha 2004). Two points are worth mentioning: (a) there is some indication that HD 170580 is He weak; (b) there is also a hint that HD 180642 is N-rich, as observed in other slowly-rotating $\beta$ Cephei stars (Morel et al. 2006), but the boron data which could be used to confirm the occurrence of deep mixing are unfortunately not available for this star.

Considering the large number of B stars in the GAUDI database and the fact that most of them are fast rotators, semi-automated spectral synthesis techniques must be developed. On the other hand, the majority of these objects are much cooler than the stars already analysed (see Fig. 1), which will require the definition of new line lists, computation of a grid of NLTE synthetic spectra down to $T_{\text {eff }} \sim 10 \mathrm{kK}$, etc. Finally, model atmosphere codes taking into account the stellar wind shall be used for the (super)giants suffering substantial mass loss. 
Table 1: Atmospheric parameters ( $\xi$ is the microturbulence velocity and $v_{T}$ is the total amount of rotational and pulsational broadening), mean NLTE abundances (on the scale in which $\log \epsilon[\mathrm{H}]=12$ ) and metallicity, $Z$, of HD 170580 and HD 180642. The number of used lines is given in brackets. The last column gives the typical abundance values found for early B dwarfs in the solar neighbourhood (Daflon \& Cunha 2004). We define $[N / C]$ and $[N / O]$ as $\log [\epsilon(N) / \epsilon(C)]$ and $\log [\epsilon(N) / \epsilon(O)]$, respectively.

\begin{tabular}{lccc}
\hline & HD 170580 & HD 180642 & B dwarfs \\
\hline$T_{\text {eff }}(\mathrm{K})$ & $20000 \pm 1000$ & $24500 \pm 1000$ & \\
$\log g([\mathrm{cgs}])$ & $4.10 \pm 0.15$ & $3.45 \pm 0.15$ & \\
$\xi\left(\mathrm{km} \mathrm{s}^{-1}\right)$ & $1_{-1}^{+5}$ & $12 \pm 3$ & \\
$v_{T}\left(\mathrm{~km} \mathrm{~s}^{-1}\right)$ & 10 & 44 & \\
$\mathrm{He} / \mathrm{H}$ & $0.048 \pm 0.021(10)$ & $0.088 \pm 0.018(4)$ & $\sim 0.085$ \\
$\log \epsilon(\mathrm{C})$ & $8.13 \pm 0.16(4)$ & $8.21 \pm 0.10(9)$ & $\sim 8.2$ \\
$\log \epsilon(\mathrm{N})$ & $7.87 \pm 0.27(14)$ & $8.00 \pm 0.19(21)$ & $\sim 7.6$ \\
$\log \epsilon(\mathrm{O})$ & $8.42 \pm 0.39(10)$ & $8.53 \pm 0.14(25)$ & $\sim 8.5$ \\
$\log \epsilon(\mathrm{Mg})$ & $7.45 \pm 0.43(1)$ & $7.34 \pm 0.20(1)$ & $\sim 7.4$ \\
$\log \epsilon(\mathrm{Al})$ & $6.23 \pm 0.24(3)$ & $6.22 \pm 0.15(3)$ & $\sim 6.1$ \\
$\log \epsilon(\mathrm{Si})$ & $7.22 \pm 0.36(7)$ & $7.19 \pm 0.19(7)$ & $\sim 7.2$ \\
$\log \epsilon(\mathrm{S})$ & $7.28 \pm 0.21(12)$ & $7.10 \pm 0.34(4)$ & $\sim 7.2$ \\
$\log \epsilon(\mathrm{Fe})$ & $7.37 \pm 0.25(9)$ & $7.34 \pm 0.21(21)$ & $\sim 7.3^{a}$ \\
$Z$ & $0.0095 \pm 0.0030$ & $0.0106 \pm 0.0016$ & $\sim 0.0099$ \\
\hline$[\mathrm{N} / \mathrm{C}]$ & $-0.26 \pm 0.32$ & $-0.21 \pm 0.22$ & $\sim-0.6$ \\
{$[\mathrm{~N} / \mathrm{O}]$} & $-0.55 \pm 0.48$ & $-0.53 \pm 0.24$ & $\sim-0.9$ \\
\hline
\end{tabular}

a Taken from Morel et al. (2006).

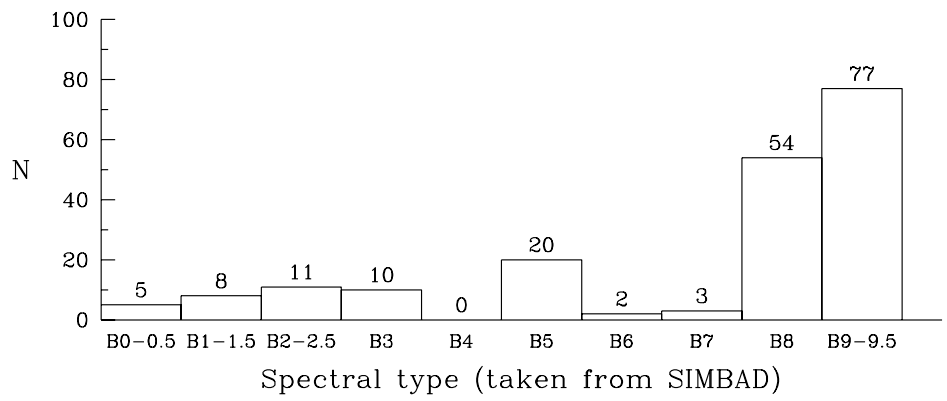

Figure 1: Breakdown by spectral type of the 190 B-type stars with spectra available in the GAUDI database at the time of writing (excluding the known Be stars).

Acknowledgments. Many thanks to Katrien Uytterhoeven for providing us with the FEROS spectra of HD 180642. T. M. acknowledges financial support from the European Space Agency through a Postdoctoral Research Fellow grant and from the Research Council of Leuven University through grant GOA/2003/04.

\section{References}

Daflon S., Cunha K., 2004, ApJ, 617, 1115

Morel T., Butler K., Aerts C., Neiner C., Briquet M., 2006, A\&A, 457, 651

Solano E., Catala C., Garrido R., et al., 2005, AJ, 129, 547 